Zhihua Wang • Libin Li

\title{
Adjoint functors raised from Ore extensions
}

Received: 5 November 2015 / Accepted: 20 July 2016 / Published online: 2 August 2016

(C) The Author(s) 2016. This article is published with open access at Springerlink.com

\begin{abstract}
Let $A$ be a $\mathbb{k}$-algebra and $A[t ; \alpha, \delta]$ its Ore extension. We give a pair of adjoint functors between the module category over $\operatorname{ker} \delta$ and the module category over $A[t ; \alpha, \delta]$. For a kind of special Ore extensions, this pair describes an equivalence between the module category over $\operatorname{ker} \delta$ and an appropriate subcategory of the module category over $A[t ; \alpha, \delta]$. Applied to the case of Weyl algebras, this is exactly a Kashiwara's theorem about $D$-modules.
\end{abstract}

Mathematics Subject Classification 13N10 $\cdot 16 \mathrm{G} 10 \cdot 16 \mathrm{U} 20$

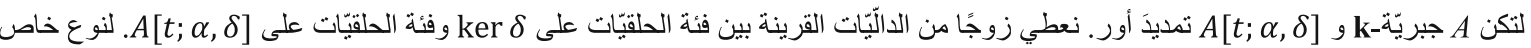

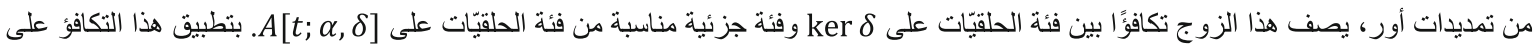

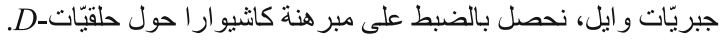

\section{Introduction}

Let $A$ be a unital associative algebra over a field $\mathbb{k}, \alpha$ a $\mathbb{k}$-linear injective endomorphism of $A$ and $\delta$ a $\mathbb{k}$-linear $\alpha$ derivation of $A$. The Ore extension of $A$ with respect to the $\alpha$-derivation $\delta$ is denoted by $B:=A[t ; \alpha, \delta]$. It is the algebra freely generated by the algebra $A$ adjoined by an indeterminate $t$ subject to the rule $t a=\alpha(a) t+\delta(a)$ for $a \in A$. These Ore extensions are very important in constructing interesting mathematical objects and hence they have received a lot of attention. We refer to [3,9] for their global dimension and Krull dimension, to $[6,10,11]$ for their ring-theoretical properties, to $[1,2,7,8]$ for their representation theory.

Observe that $A^{\delta}:=\operatorname{ker} \delta$ is a subalgebra of $A$ and hence $A$ is a right $A^{\delta}$-module under multiplication. The algebra homomorphism from $B$ to $\operatorname{End}_{\mathbb{k}}(A)$ given by $\sum_{i \geq 0} a_{i} t^{i} \mapsto \sum_{i \geq 0} a_{i} \delta^{i}$ (see e.g., [5]) induces a left $B$-module structure on $A$. Moreover, it is easy to see that $A$ is a $B$ - $A^{\delta}$-bimodule. This leads to a functor $A \otimes_{A^{\delta-}}$ from the category of left $A^{\delta}$-modules into the category of left $B$-modules. In addition, if $X$ is a left $B$-module, then the $\mathbb{k}$-subspace of $X \operatorname{ker}_{t} X:=\{\mu \in X \mid t \cdot \mu=0\}$ is a left $A^{\delta}$-module. So we obtain a functor $\mathrm{ker}_{t^{-}}$ from the category of left $B$-modules to that of left $A^{\delta}$-modules.

The aim of the paper is to study the relationship between the functors $A \otimes_{A^{\delta}}$ and $\operatorname{ker}_{t^{-}}$. We show that these functors form an adjoint pair of functors. Moreover, the pair gives an equivalence of appropriate categories if

\section{Z. Wang $(\varangle)$}

Department of Mathematical Sciences, Taizhou University, Taizhou 225300, People's Republic of China

E-mail: mailzhihua@126.com

L. Li

School of Mathematical Science, Yangzhou University, Yangzhou 225002, People's Republic of China

E-mail: lbli@yzu.edu.cn 
the Ore extension satisfies some special conditions. The paper is organized as follows. In Sect. 2, we first set the notations and present some basic results on Ore extensions. Then we show that the functor $A \otimes_{A^{\delta}}$ is left adjoint to the functor $\mathrm{ker}_{t^{-}}$. This leads to a result that the endomorphism algebra $\operatorname{End}_{B}\left(A \otimes_{A^{\delta}} M\right)$ is isomorphic to $\operatorname{End}_{A^{\delta}}(M)$ if $A^{\delta}$-module $M$ satisfies an additional property. In this case, $M$ is an indecomposable $A^{\delta}$-module if and only if $A \otimes_{A^{\delta}} M$ is an indecomposable $B$-module.

In Sect. 3, we first show that the set $T=\left\{t^{i} \mid i \geq 0\right\}$ is a left Ore set of $B$ with the assumption that the $\alpha$-derivation $\delta$ is locally nilpotent. Then any simple $B$-module is either $T$-torsion or $T$-torsion free. We use the functor ker $_{t}$ - to characterize when a simple $B$-module is $T$-torsion or $T$-torsion free.

Obviously, the adjoint pair given above do not in general describe an equivalence of categories. In Sect. 4, we consider a class of Ore extensions and, if we restrict to an appropriate subcategory of $B$-module category, then the adjoint pair do restrict to an equivalence. In case where the Ore extension algebras are Weyl algebras, this gives rise to a Kashiwara's theorem, one of the major results of the theory of $D$-modules.

In Sect. 5, we deal with particular Ore extension algebras, i.e., the $n$th Weyl algebras. We mainly describe the relationship of multiplicity as well as Gelfand-Kirillov dimension between finitely generated $A^{\delta}$-module $M$ and the induced $B$-module $A \otimes_{A^{\delta}} M$. This leads to a result that $M$ is a holonomic $A^{\delta}$-module if and only if $A \otimes_{A^{\delta}} M$ is a holonomic $B$-module.

\section{The adjunction of functors}

In this section, we give a description of the functor $A \otimes_{A^{\delta}}$ as well as $\operatorname{ker}_{t^{-}}$. We show that the functor $A \otimes_{A^{\delta-}}$ is left adjoint to the functor ker $_{t^{-}}$. This leads to an algebra isomorphism from the endomorphism algebra $\operatorname{End}_{B}\left(A \otimes_{A^{\delta}} M\right)$ to $\operatorname{End}_{A^{\delta}}(M)$ if the $A^{\delta}$-module $M$ satisfies a special condition. In this case, the property of indecomposability of the $A^{\delta}$-module $M$ can be lifted to the induced $B$-module $A \otimes_{A^{\delta}} M$.

In the following, $A$ is a unital associative algebra over a field $\mathbb{k}$ and $\alpha$ is an injective endomorphism of $A$. The $\mathbb{k}$-linear map $\delta$ from $A$ to itself is called an $\alpha$-derivation of $A$ if $\delta(a b)=\alpha(a) \delta(b)+\delta(a) b$, for any $a, b \in A$. The $\alpha$-derivation $\delta$ is called locally nilpotent if for any $a \in A$, there exists some natural number $n$ such that $\delta^{n}(a)=0$. The Ore extension of $A$ with respect to the $\alpha$-derivation $\delta$ is a unital associative algebra $B:=A[t ; \alpha, \delta]$, where $B$ is generated as an algebra by the indeterminate $t$ over $A$ subject to

$$
t a=\alpha(a) t+\delta(a), \quad \text { for } a \in A .
$$

It can be seen by induction on $n \geq 1$ that

$$
t^{n} a=\alpha^{n}(a) t^{n}+a_{n-1} t^{n-1}+\cdots+a_{1} t+\delta^{n}(a), \quad \text { for } a, a_{1}, \ldots, a_{n-1} \in A .
$$

The algebra $B$ is a free left $A$-module with an $A$-basis $\left\{t^{i} \mid i \geq 0\right\}$. If $\alpha$ is an automorphism of $A$, then $B$ is also a free right $A$-module. Note that

$$
B \rightarrow \operatorname{End}_{\mathbb{k}}(A), \quad \sum_{i \geq 0} a_{i} t^{i} \mapsto \sum_{i \geq 0} a_{i} \delta^{i}
$$

is an algebra homomorphism [5]. This induces a left $B$-module structure on $A$ defined by

$$
\left(\sum_{i \geq 0} a_{i} t^{i}\right) \cdot a=\sum_{i \geq 0} a_{i} \delta^{i}(a), \quad \text { for } a_{i}, a \in A .
$$

Denote by $A^{\delta}:=\operatorname{ker} \delta$. Then $A^{\delta}$ is a subalgebra of $A$. Consider $A$ as a right $A^{\delta}$-module, where the action is given by multiplication. Then $A$ is a $B$ - $A^{\delta}$-bimodule, since the relation $\delta^{i}(b a)=\delta^{i}(b) a$ implies that

$$
\left(\left(\sum_{i \geq 0} a_{i} t^{i}\right) \cdot b\right) a=\left(\sum_{i \geq 0} a_{i} t^{i}\right) \cdot(b a), \quad \text { for } b \in A \quad \text { and } \quad a, a_{i} \in A^{\delta} .
$$

Let $A^{\delta}$-Mod and $B$-Mod denote the left module categories over $A^{\delta}$ and $B$, respectively. If $X$ is a left $B$-module, it follows from (2.1) that the $\mathbb{k}$-subspace $\operatorname{ker}_{t} X:=\{\mu \in X \mid t \cdot \mu=0\}$ of $X$ is a left $A^{\delta}$-module. In particular, for any $B$-module morphism $g: X \rightarrow Y$, we have $g\left(\operatorname{ker}_{t} X\right) \subseteq \operatorname{ker}_{t} Y$. Thus, the restriction of $g$ to the subspace $\operatorname{ker}_{t} X$ of $X$ gives rise to the morphism $\operatorname{ker}_{t} g: \operatorname{ker}_{t} X \rightarrow \operatorname{ker}_{t} Y$. It is obvious that $\operatorname{ker}_{t}$ - is an additive $\mathbb{k}$-linear covariant left exact functors from $B$-Mod to $A^{\delta}$-Mod. 
Proposition 2.1 We have the following descriptions of the functors $\operatorname{ker}_{t^{-}}$and $A \otimes_{A^{\delta-}}$ :

(1) For any $B$-module $X, \operatorname{Hom}_{B}(A, X)$ is isomorphic to $\operatorname{ker}_{t} X$ as $A^{\delta}$-modules.

(2) There is a natural equivalence from the functor $\operatorname{Hom}_{B}(A,-)$ to $\operatorname{ker}_{t}$-.

(3) The functor $A \otimes_{A^{\delta}-}$ is left adjoint to $\mathrm{ker}_{t^{-}}$, or equivalently, $\mathrm{ker}_{t^{-}}$is right adjoint to $A \otimes_{A^{\delta}}$.. Namely, there exists an isomorphism

$$
\operatorname{Hom}_{B}\left(A \otimes_{A^{\delta}} M, X\right) \cong \operatorname{Hom}_{A^{\delta}}\left(M, \operatorname{ker}_{t} X\right)
$$

for any $A^{\delta}$-module $M$ and $B$-module $X$, which is functorial at $M$ and $X$.

Proof (1) Note that $\operatorname{Hom}_{B}(A, X)$ admits a left $A^{\delta}$-module structure determined by $(a \cdot f)(b)=f(b a)$, for any $a \in A^{\delta}, f \in \operatorname{Hom}_{B}(A, X)$ and $b \in A$. We define the following two $\mathbb{k}$-linear maps:

$$
\varphi_{X}: \operatorname{Hom}_{B}(A, X) \rightarrow \operatorname{ker}_{t} X, \quad f \mapsto f(1)
$$

and

$$
\psi_{X}: \operatorname{ker}_{t} X \rightarrow \operatorname{Hom}_{B}(A, X) \quad \mu \mapsto \psi_{X}(\mu): b \mapsto b \cdot \mu .
$$

We have $f(1) \in \operatorname{ker}_{t} X$ since $t \cdot f(1)=f(t \cdot 1)=f(\delta(1))=0$. To verify that $\psi_{X}(\mu) \in \operatorname{Hom}_{B}(A, X)$, we only need to check that $\psi_{X}(\mu)(t \cdot b)=t \cdot \psi_{X}(\mu)(b)$ for $b \in A$ since the map $\psi_{X}(\mu)$ is obvious A-linearly. Indeed,

$$
\begin{aligned}
t \cdot \psi_{X}(\mu)(b) & =t \cdot(b \cdot \mu)=(t b) \cdot \mu=(\alpha(b) t+\delta(b)) \cdot \mu \\
& =\delta(b) \cdot \mu=\psi_{X}(\mu)(\delta(b))=\psi_{X}(\mu)(t \cdot b) .
\end{aligned}
$$

It is straightforward to show that $\psi_{X} \circ \varphi_{X}=\operatorname{id}_{\operatorname{Hom}_{B}(A, X)}$ and $\varphi_{X} \circ \psi_{X}=\operatorname{id}_{\operatorname{ker}_{t} X}$. The rest is to check that $\varphi_{X}$ and $\psi_{X}$ are both $A^{\delta}$-linear. In fact, for any $a \in A^{\delta}$ and $f \in \operatorname{Hom}_{B}(A, X)$, the $A^{\delta}$-module structure on $\operatorname{Hom}_{B}(A, X)$ implies that

$$
\varphi_{X}(a \cdot f)=(a \cdot f)(1)=f(1 a)=f(a 1)=a \cdot f(1)=a \cdot \varphi_{X}(f) .
$$

On one hand we have, for any $b \in A, \psi_{X}(a \cdot \mu)(b)=b \cdot(a \cdot \mu)=(b a) \cdot \mu$, and on the other hand since $\psi_{X}(\mu) \in \operatorname{Hom}_{B}(A, X)$ and $\operatorname{Hom}_{B}(A, X)$ is an $A^{\delta}$-module, we have $\left(a \cdot \psi_{X}(\mu)\right)(b)=\psi_{X}(\mu)(b a)=$ $(b a) \cdot \mu$. We obtain the desired result.

(2) For any $B$-module morphism $f: X \rightarrow Y$, we have $A^{\delta}$-module isomorphisms $\varphi_{X}: \operatorname{Hom}_{B}(A, X) \rightarrow$ $\operatorname{ker}_{t} X$ and $\varphi_{Y}: \operatorname{Hom}_{B}(A, Y) \rightarrow \operatorname{ker}_{t} Y$ by Part (1). It is direct to check that the diagram

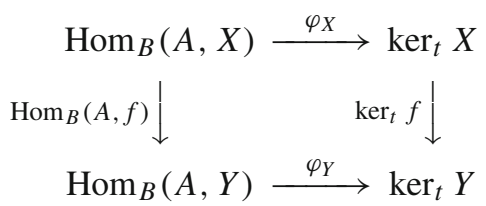

is commutative in $A^{\delta}$-Mod. Thus, $\varphi=\left\{\varphi_{X}\right\}_{X \in B \text {-Mod }}$ gives a natural equivalence from the functor $\operatorname{Hom}_{B}(A,-)$ to $\operatorname{ker}_{t^{-}}$.

(3) Note that the functor $A \otimes_{A^{\delta}}$ - is left adjoint to $\operatorname{Hom}_{B}(A,-)$; that is, there is an isomorphism

$$
\tau: \operatorname{Hom}_{B}\left(A \otimes_{A^{\delta}} M, X\right) \rightarrow \operatorname{Hom}_{A^{\delta}}\left(M, \operatorname{Hom}_{B}(A, X)\right), f \mapsto \tau_{f},
$$

which is functorial at $A^{\delta}$-module $M$ and $B$-module $X$. The morphism $\tau_{f}$ is given by $\tau_{f}(m)(a)=f(a \otimes m)$, for any $m \in M$ and $a \in A$. The isomorphism $\varphi_{X}: \operatorname{Hom}_{B}(A, X) \rightarrow \operatorname{ker}_{t} X, f \mapsto f(1)$ induces an isomorphism

$$
\operatorname{Hom}_{A^{\delta}}\left(M, \varphi_{X}\right): \operatorname{Hom}_{A^{\delta}}\left(M, \operatorname{Hom}_{B}(A, X)\right) \rightarrow \operatorname{Hom}_{A^{\delta}}\left(M, \operatorname{ker}_{t} X\right) .
$$

Then the following composition

$$
\operatorname{Hom}_{A^{\delta}}\left(M, \varphi_{X}\right) \circ \tau: \operatorname{Hom}_{B}\left(A \otimes_{A^{\delta}} M, X\right) \rightarrow \operatorname{Hom}_{A^{\delta}}\left(M, \operatorname{ker}_{t} X\right)
$$

gives rise to a desired isomorphism which is functorial at $M$ and $X$. 
According to Proposition 2.1, we are able to characterize the indecomposability and simplicity of $B$-module $A$ as shown in the following.

Corollary 2.2 For the B-module A, we have the following:

(1) A is indecomposable if and only if the subalgebra $A^{\delta}$ of A contains only the trivial idempotents.

(2) If the $\alpha$-derivation $\delta$ is locally nilpotent, then $A$ is simple if and only if the subalgebra $A^{\delta}$ of $A$ is a division algebra.

Proof By replacing the $B$-module $X$ with $A$ in Proposition 2.1 (1), we obtain an algebra isomorphism from the endomorphism algebra $\operatorname{End}_{B}(A)$ to the opposite algebra $\left(A^{\delta}\right)^{\text {op }}$ of $A^{\delta}$. Thus, Part (1) and the necessity of Part (2) follow. To prove the sufficiency of Part (2), we suppose that $I$ is a non-zero submodule of $A$. Then $I$ is a left ideal of $A$ satisfying $\delta(I) \subseteq I$. For any non-zero element $a \in I$, there exists $n \geq 0$ such that $\delta^{n+1}(a)=0$ while $\delta^{n}(a) \neq 0$ since the derivation $\delta$ is locally nilpotent. This implies that $\delta^{n}(a) \in A^{\delta} \cap I$. The condition $A^{\delta}$ is a division algebra which implies that $\delta^{n}(a)$ is invertible, so we obtain an invertible element $\delta^{n}(a)$ in the left ideal $I$ of $A$, this yields that $I=A$.

If there is an $A^{\delta}$-module isomorphism

$$
\theta: \operatorname{ker}_{t}\left(A \otimes_{A^{\delta}} M\right) \rightarrow M, 1 \otimes m \mapsto m .
$$

Then the property of indecomposability of the $A^{\delta}$-module $M$ can be lifted to the induced $B$-module $A \otimes_{A^{\delta}} M$.

Theorem 2.3 Let $M$ be an $A^{\delta}$-module satisfying the condition (2.6). Then the endomorphism algebra $\operatorname{End}_{B}\left(A \otimes_{A^{\delta}} M\right)$ is isomorphic to $\operatorname{End}_{A^{\delta}}(M)$. In this case, $M$ is an indecomposable $A^{\delta}$-module if and only if $A \otimes_{A^{\delta}} M$ is an indecomposable $B$-module.

Proof Note that the isomorphism $\theta: \operatorname{ker}_{t}\left(A \otimes_{A^{\delta}} M\right) \rightarrow M$ induces the isomorphism

$$
\operatorname{Hom}_{A^{\delta}}(M, \theta): \operatorname{Hom}_{A^{\delta}}\left(M, \operatorname{ker}_{t} A \otimes_{A^{\delta}} M\right) \rightarrow \operatorname{End}_{A^{\delta}}(M) .
$$

By replacing $B$-module $X$ with $A \otimes_{A^{\delta}} M$ in (2.5), we obtain the isomorphism

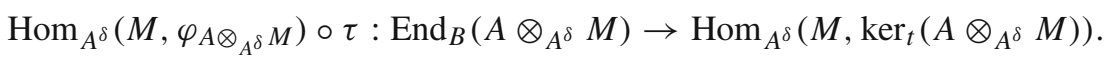

The composition of the two isomorphisms stated above is denoted by

$$
\Gamma_{M}:=\operatorname{Hom}_{A^{\delta}}(M, \theta) \circ \operatorname{Hom}_{A^{\delta}}\left(M, \varphi_{A \otimes_{A^{\delta}} M}\right) \circ \tau .
$$

Then $\Gamma_{M}$ is an isomorphism from $\operatorname{End}_{B}\left(A \otimes_{A^{\delta}} M\right)$ to $\operatorname{End}_{A^{\delta}}(M)$. In the following, we shall verify that this isomorphism respects the algebra structure. For any $g \in \operatorname{End}_{B}\left(A \otimes_{A^{\delta}} M\right)$ and $m \in M$, we have $g(1 \otimes m) \in$ $\operatorname{ker}_{t}\left(A \otimes_{A^{\delta}} M\right)$ since $t \cdot g(1 \otimes m)=g(t \cdot(1 \otimes m))=g(\delta(1) \otimes m)=0$. It follows that $\theta(g(1 \otimes m)) \in M$. By the requirement of the map $\theta$, we have $\theta(1 \otimes \theta(g(1 \otimes m)))=\theta(g(1 \otimes m))$, and hence

$$
1 \otimes \theta(g(1 \otimes m))=g(1 \otimes m) .
$$

With this preparation, we have

$$
\begin{aligned}
& \Gamma_{M}(g)(m)=\left(\operatorname{Hom}_{A^{\delta}}(M, \theta) \circ \operatorname{Hom}_{A^{\delta}}\left(M, \varphi_{A \otimes_{A^{\delta}} M}\right) \circ \tau\right)(g)(m) \\
& =\left(\theta \circ \varphi_{A \otimes_{A^{\delta}} M} \circ \tau_{g}\right)(m) \\
& =\theta\left(\tau_{g}(m)(1)\right) \\
& =\theta(g(1 \otimes m)) \text {. }
\end{aligned}
$$

If $g=\mathrm{id}$, the identity of $\operatorname{End}_{B}\left(A \otimes_{A^{\delta}} M\right)$, then the Eq. (2.8) becomes

$$
\Gamma_{M}(\mathrm{id})(m)=\theta(1 \otimes m)=m \text {. }
$$

That is, $\Gamma_{M}$ (id) is the identity of $\operatorname{End}_{A^{\delta}}(M)$. For any $f \in \operatorname{End}_{B}\left(A \otimes_{A^{\delta}} M\right)$, by (2.8), we have

$$
\begin{aligned}
\left(\Gamma_{M}(f) \circ \Gamma_{M}(g)\right)(m) & =\Gamma_{M}(f)(\theta(g(1 \otimes m))) \\
& =\theta(f(1 \otimes \theta(g(1 \otimes m)))) \\
& =\theta(f(g(1 \otimes m))) \text { by }(2.7) \\
& =\Gamma_{M}(f \circ g)(m) .
\end{aligned}
$$

We conclude that $\Gamma_{M}$ is an algebra isomorphism from $\operatorname{End}_{B}\left(A \otimes_{A^{\delta}} M\right)$ to $\operatorname{End}_{A^{\delta}}(M)$. Thus, $M$ is an indecomposable $A^{\delta}$-module if and only if $A \otimes_{A^{\delta}} M$ is an indecomposable $B$-module. 
The condition (2.6) looks rather strong. However, as we shall see that it is quite often satisfied.

Proposition 2.4 Let $A$ be a free right $A^{\delta}$-module with a basis $\left\{x_{i} \mid i \geq 0\right\}$ with $x_{0}=1$. Then any left ideal I of $A^{\delta}$ satisfies the condition (2.6).

Proof We first verify that $\left\{\delta\left(x_{i}\right) \mid i \geq 1\right\}$ is right $A^{\delta}$-linearly independent. If $\sum_{i \geq 1} \delta\left(x_{i}\right) a_{i}=0$, for some $a_{i} \in$ $A^{\delta}$, then $\delta\left(\sum_{i \geq 1} x_{i} a_{i}\right)=\sum_{i \geq 1} \delta\left(x_{i}\right) a_{i}=0$. It follows that $\sum_{i \geq 1} x_{i} a_{i} \in A^{\delta}$. Denote by $a_{0}=-\sum_{i \geq 1} x_{i} a_{i}$. Then $\sum_{i \geq 0} x_{i} a_{i}=0$. This implies that $a_{i}=0$ for any $i \geq 0$ since $\left\{x_{i} \mid i \geq 0\right\}$ is a basis of $A$ as a free right $A^{\delta}$-module. Now it is direct to calculate that $\operatorname{ker}_{t}\left(A \otimes_{A^{\delta}} I\right)=\{1 \otimes m \mid m \in I\}$ and the map

$$
I \rightarrow \operatorname{ker}_{t}\left(A \otimes_{A^{\delta}} I\right), m \mapsto 1 \otimes m
$$

is an $A^{\delta}$-module isomorphism.

Example 2.5 Let $A=\mathbb{k}[x]$ be a polynomial algebra over a field $\mathbb{k}, q \in \mathbb{k} \backslash\{0,1\}$, and $\alpha$ an automorphism of $A$ satisfying $\alpha(x)=q x$. Then the formula

$$
\delta(f(x))=\frac{f(q x)-f(x)}{q x-x}=\frac{\alpha(f(x))-f(x)}{\alpha(x)-x}
$$

defines an $\alpha$-derivation on $A$. This derivation is locally nilpotent and is known as the $q$-difference operator or Eulerian derivation. Let $B=A[t ; \alpha, \delta]$. Then $B$ is known as a quantized Weyl algebra over $\mathbb{k}$. It is obvious that

$$
A^{\delta}= \begin{cases}\mathbb{k}, & \mathrm{q} \text { is not a root of unity, } \\ \mathbb{k}\left[x^{n}\right], & \mathrm{q} \text { is a } n \text {th root of unity. }\end{cases}
$$

Following from Corollary 2.2 (2), we obtain that $A$ is a simple $B$-module if $q$ is not a root of unity. If $q$ is a $n$th root of unity, then $A$ is a free $A^{\delta}$-module with a basis $\left\{1, x, \ldots, x^{n-1}\right\}$. In this case, any $A^{\delta}$-module $M$ satisfies the condition (2.6) since the map $M \rightarrow \operatorname{ker}_{t}\left(A \otimes_{A^{\delta}} M\right), m \mapsto 1 \otimes m$ is an $A^{\delta}$-module isomorphism.

\section{Ore quotient rings and modules}

In this section, we always assume that the $\alpha$-derivation $\delta$ in $B=A[t ; \alpha, \delta]$ is locally nilpotent. In this case, the set $T=\left\{t^{i} \mid i \geq 0\right\}$ is a left Ore set of $B$. The aim of this section is to characterize simple $T$-torsion as well as simple $T$-torsion-free modules over $B$ through the functor $\operatorname{ker}_{t^{-}}$.

Definition 3.1 A set $D$ in a ring $R$ is called a left Ore set of $R$ if the following two conditions are satisfied:

(1) $D$ is a multiplicative set of $R$, that is, the unity $1 \in D$ and for any two $a, b \in D, a b \in D$;

(2) For any $a \in D$ and $r \in R$, there exist $b \in D$ and $s \in R$ such that $b r=s a$, that is, $D r \cap R a \neq \emptyset$.

A right Ore set of ring $R$ is defined as a left Ore set of opposite ring $R^{\text {op }}$, and an Ore set of $R$ is simultaneously left and right Ore set, see [5] for details.

Proposition 3.2 The set $T=\left\{t^{i} \mid i \geq 0\right\}$ is a left Ore set of the algebra $B=A[t ; \alpha, \delta]$.

Proof We assert that for any $a \in B$ and $t^{i} \in T$, there exist $b \in B$ and $t^{j} \in T$ such that $t^{j} a=b t^{i}$. To this end, we denote by $a=b_{0} t+a_{0}$, for some $b_{0} \in B$ and $a_{0} \in A$. Since the derivation $\delta$ is locally nilpotent, there exists $n_{1} \geq 0$ such that $\delta^{n_{1}}\left(a_{0}\right)=0$. It follows from (2.2) that $t^{n_{1}} a_{0}=b_{1} t$, for some $b_{1} \in B$. Hence,

$$
t^{n_{1}} a=t^{n_{1}}\left(b_{0} t+a_{0}\right)=\left(t^{n_{1}} b_{0}+b_{1}\right) t=a_{1} t .
$$

Similarly, for $a_{1} \in B$, there exist $n_{2} \geq 0$ and $a_{2} \in B$ such that $t^{n_{2}} a_{1}=a_{2} t$. Thus, $t^{n_{1}+n_{2}} a=a_{2} t^{2}$. Repeating the argument, we obtain that $t^{n_{1}+\cdots+n_{i}} a=a_{i} t^{i}$, for some $a_{i} \in B$, as required.

According to Proposition 3.2, we have the following corollary.

Corollary 3.3 For any $B$-module $X$, the subset

$$
\operatorname{tor}_{T}(X)=\{\mu \in X \mid \mu \text { is annihilated by a power of } t\}
$$

is a submodule of $X$. 
Definition 3.4 For any $B$-module $X$, if $\operatorname{tor}_{T}(X)=X$ then $X$ is called $T$-torsion, if tor $(X)=0$ then $X$ is called $T$-torsion free.

Proposition 3.5 We have the following:

(1) The regular module $B$ is $T$-torsion free. Moreover, any free module over $B$ is $T$-torsion free.

(2) Any flat module over $B$ is $T$-torsion free.

Proof (1) It is straightforward to check using the definition of a $T$-torsion-free module.

(2) Let $X$ be a flat $B$-module. Then there exists an epimorphism $\phi: F \rightarrow X$, where $F$ is a free $B$-module. For any $\mu \in \operatorname{tor}_{T}(X)$, there is $t^{i} \in T$ such that $t^{i} \mu=0$, and $v \in F$ such that $\phi(v)=\mu$. Then $t^{i} v \in \operatorname{ker} \phi$ since $\phi\left(t^{i} v\right)=t^{i} \phi(v)=0$. Consider $t^{i} B$ as a right ideal of $B$, then $t^{i} B F \cap \operatorname{ker} \phi=t^{i} B \operatorname{ker} \phi$ since $X$ is flat. Note that $t^{i} v \in t^{i} B F \cap \operatorname{ker} \phi=t^{i} B \operatorname{ker} \phi$. We have $t^{i} v=\sum_{j} t^{i} b_{j} v_{j}$, for some $b_{j} \in B$ and $v_{j} \in \operatorname{ker} \phi$. Then $t^{i}\left(v-\sum_{j} b_{j} v_{j}\right)=0$, and hence $v-\sum_{j} b_{j} v_{j} \in \operatorname{tor}_{T}(F)=0$. It follows that $v=\sum_{j} b_{j} v_{j} \in \operatorname{ker} \phi$, and hence $\mu=\phi(v)=0$.

We are ready to consider the structure of $T^{-1} B$, the left Ore localization of the ring $B$ with respect to the left Ore set $T$, see [5] for a complete introduction. Let the symbol $\sim$ be an equivalence relation on $B \times T$ defined by $\left(a, t^{i}\right) \sim\left(b, t^{j}\right), i \geq j$ if and only if there exist $k, l \geq 0$ such that $t^{k} a=t^{l} b$ and $t^{k} t^{i}=t^{l} t^{j}$, this is precisely $a=t^{i-j} b$. The equivalence class of any pair $\left(a, t^{i}\right)$ in $B \times T$ is denoted by $\frac{a}{t^{i}}$, and the set of these equivalence classes is denoted by $T^{-1} B$.

The addition and multiplication of elements $\frac{a}{t^{i}}$ and $\frac{b}{t^{j}}, i \geq j$ in $T^{-1} B$ are defined as follows:

- $\frac{a}{t^{i}}+\frac{b}{t^{j}}=\frac{a+t^{i-j} b}{t^{i}}$.

- $\frac{a}{t^{i}} \cdot \frac{b}{t^{j}}=\frac{c b}{t^{i+k}}$, where $c \in B$ and $t^{k} \in T$ such that $t^{k} a=c t^{j}$.

It can be seen from these definitions that $T^{-1} B$ is equipped with a unital ring structure. The natural ring morphism $B \rightarrow T^{-1} B, a \mapsto \frac{a}{1}$ is injective since $\left(a, t^{i}\right) \sim(0,1)$ if and only if $a=0$.

Having constructed the left ring of fractions $T^{-1} B$ with respect to $T$, we return to the construction of an analogous module of fraction $T^{-1} X$ for any left $B$-module $X$. Define an equivalence relation $\sim$ on $X \times T$ as $\left(\mu, t^{i}\right) \sim\left(\nu, t^{j}\right), i \geq j$ if and only if there exist $k, l \geq 0$ such that $t^{k} \mu=t^{l} v$ and $t^{k} t^{i}=t^{l} t^{j}$, this is precisely $\mu=t^{i-j} v$ provided that $\operatorname{ker}_{t} X=0$. The equivalence class of any pair $\left(\mu, t^{i}\right)$ in $X \times T$ is denoted by $\frac{\mu}{t^{i}}$ and the set of these equivalence classes is denoted by $T^{-1} X$.

$T^{-1} X$ is a left $T^{-1} B$-module, where the $T^{-1} B$-module structure on $T^{-1} X$ is given as follows: for any $\frac{a}{t^{i}}$ in $T^{-1} B$ and $\frac{\mu}{t^{i}}, \frac{v}{t^{j}}, i \geq j$ in $T^{-1} B$,

- $\frac{\mu}{t^{i}}+\frac{v}{t^{j}}=\frac{\mu+t^{i-j} v}{t^{i}}$.

- $\frac{a}{t^{i}} \cdot \frac{v}{t^{j}}=\frac{c v}{t^{i+k}}$, where $c \in B$ and $t^{k} \in T$ such that $t^{k} a=c t^{j}$.

The natural $T^{-1} B$-module morphism $X \rightarrow T^{-1} X, \mu \mapsto \frac{\mu}{1}$ is not injective in general, since $\left(\mu, t^{i}\right) \sim$ $(0,1)$ if and only if $t^{k} \mu=0$, which does not mean that $\mu=0$. However, if $\operatorname{ker}_{t} X=0$, then the natural morphism is injective. We have the following descriptions of any simple $T$-torsion $B$-module.

Theorem 3.6 Let $X$ be a simple B-module. The following are equivalent:

(1) $T^{-1} X=0$.

(2) $\operatorname{ker}_{t} X \neq 0$.

(3) $X$ is $T$-torsion.

Proof (1) $\Rightarrow$ (2) If $T^{-1} X=0$, then $\operatorname{ker}_{t} X \neq 0$. Otherwise, the natural $T^{-1} B$-module morphism $X \rightarrow$ $T^{-1} X, \mu \mapsto \frac{\mu}{1}$ is injective, a contradiction.

(2) $\Rightarrow$ (3) Note that $\operatorname{ker}_{t} X \subseteq \operatorname{tor}_{T}(X) \subseteq X$. If $\operatorname{ker}_{t} X \neq 0$, then $X=\operatorname{tor}_{T}(X)$ by the simplicity of $X$.

(3) $\Rightarrow$ (1) Note that $T^{-1} X$ is isomorphic to $T^{-1} B \otimes_{B} X$ as $T^{-1} B$-modules [5, Proposition 10.12]. We assert that $T^{-1} B \otimes_{B} X=0$ with the assumption that $X=\operatorname{tor}_{T}(X)$. Indeed, for any $\sum_{i} \frac{b_{i}}{t^{i}} \otimes \mu_{i} \in T^{-1} B \otimes_{B} X$, 
$\mu_{i} \in X=\operatorname{tor}_{T}(X)$ implies that there exists $t^{k_{i}} \in T$ such that $t^{k_{i}} \mu_{i}=0$. For each $b_{i} \in B$, there exist $t^{m_{i}} \in T$ and $b_{i}^{\prime} \in B$ such that $t^{m_{i}} b_{i}=b_{i}^{\prime} t^{k_{i}}$. We obtain that

$$
\sum_{i} \frac{b_{i}}{t^{i}} \otimes \mu_{i}=\sum_{i} \frac{t^{m_{i}} b_{i}}{t^{m_{i}} t^{i}} \otimes \mu_{i}=\sum_{i} \frac{b_{i}^{\prime} t^{k_{i}}}{t^{m_{i}+i}} \otimes \mu_{i}=\sum_{i} \frac{b_{i}^{\prime}}{t^{m_{i}+i}} \otimes t^{k_{i}} \mu_{i}=0,
$$

as desired.

For any simple $B$-module $X, X$ is either $T$-torsion or $T$-torsion free. By the observation of Theorem 3.6, we have the following corollary.

Corollary 3.7 Let $X$ be a simple B-module. The following are equivalent:

(1) $T^{-1} X \neq 0$.

(2) $\operatorname{ker}_{t} X=0$.

(3) $X$ is $T$-torsionfree.

\section{Kashiwara-like theorem}

In this section, the field $\mathbb{k}$ is of characteristic zero, $\mathbb{k}^{\times}:=\mathbb{k} \backslash\{0\}$. We assume that there exists $x \in A$ such that $\left\{x^{i} \mid i \geq 0\right\}$ forms a basis of $A$ as a free right $A^{\delta}$-module. We also assume that $\delta^{i}\left(x^{i}\right) \in \mathbb{k}^{\times}$for $i \geq 0$, and the restriction of $\alpha$ to the subalgebra $A^{\delta}$ of $A$ is an automorphism, whose inverse map is denoted by $\alpha^{-1}$ if there is no confusion. Typical examples of such Ore extensions include the $n$th Weyl algebras for $n>1$ [4], quantized Weyl algebras as described in Example 2.5 for $q$ not a root of unity.

The aim of this section is to give the so-called Kashiwara-like theorem for this kind of Ore extension algebras. If the Ore extension algebras are specialized to Weyl algebras, we recover the well-known Kashiwara's theorem.

Lemma 4.1 We have the following description of $A$ :

(1) $A=A^{\delta}\left[x ; \alpha^{-1}, \gamma\right]$, where the $\alpha^{-1}$-derivation $\gamma$ of $A^{\delta}$ is given by $\gamma(a)=x a-\alpha^{-1}(a) x$ for all $a \in A^{\delta}$.

(2) $\alpha(x)=\varepsilon x+a_{0}$, for some $a_{0} \in A^{\delta}$ and $\varepsilon \in \mathbb{k}^{\times}$which is not a root of unity.

Proof (1) Note that $\delta(\gamma(a))=\delta\left(x a-\alpha^{-1}(a) x\right)=\delta(x) a-a \delta(x)=0$ for any $a \in A^{\delta}$. It follows that $\gamma(a) \in A^{\delta}$ and $\gamma$ is a $\mathbb{k}$-linear map of $A^{\delta}$. For any $a, b \in A^{\delta}$,

$$
\begin{aligned}
\gamma(a b) & =x a b-\alpha^{-1}(a b) x \\
& =\alpha^{-1}(a) x b-\alpha^{-1}(a b) x+x a b-\alpha^{-1}(a) x b \\
& =\alpha^{-1}(a)\left(x b-\alpha^{-1}(b) x\right)+\left(x a-\alpha^{-1}(a) x\right) b \\
& =\alpha^{-1}(a) \gamma(b)+\gamma(a) b .
\end{aligned}
$$

Thus, $\gamma$ is an $\alpha^{-1}$-derivation of $A^{\delta}$. Since $A$ is a free right $A^{\delta}$-module with a basis $\left\{x^{i} \mid i \geq 0\right\}, A$ is equal to $A^{\delta}\left[x ; \alpha^{-1}, \gamma\right]$ as right $A^{\delta}$-modules. Moreover, $A$ and $A^{\delta}\left[x ; \alpha^{-1}, \gamma\right]$ are isomorphic as $\mathbb{k}$-algebras since their multiplication rules coincide.

(2) Note that $\delta^{2}\left(x^{2}\right)=\alpha^{2}(x) \delta^{2}(x)+\delta(\alpha(x)) \delta(x)+\alpha(\delta(x)) \delta(x)+\delta^{2}(x) x$. Then the condition $\delta^{i}\left(x^{i}\right) \in \mathbb{k}^{\times}$ implies that $\delta(\alpha(x)) \in \mathbb{k}^{\times}$. We assume that $\alpha(x)=\sum_{i=0}^{n} a_{i} x^{i}$, for $a_{i} \in A^{\delta}$ with $a_{n} \neq 0$. If $n>1$, then $\delta^{n}(\alpha(x))=0$. However, $\delta^{n}(\alpha(x))=\alpha^{n}\left(a_{n}\right) \delta^{n}\left(x^{n}\right)$. It follows that $\alpha^{n}\left(a_{n}\right)=0$, a contradiction to $a_{n} \neq 0$. Thus, $n=1$ and hence $\alpha(x)=a_{0}+a_{1} x$. In this case, $\delta(\alpha(x))=\alpha\left(a_{1}\right) \delta(x)$. Then $\delta(\alpha(x)) \in \mathbb{k}^{\times}$implies that $\alpha\left(a_{1}\right) \in \mathbb{k}^{\times}$, and hence $a_{1} \in \mathbb{k}^{\times}$. We assert that $\varepsilon:=a_{1}$ is not a root of unity. Indeed, by induction on $n$, we obtain that the leading term $\lambda_{n-1} x^{n-1}$ of $\delta\left(x^{n}\right)$ is equal to $\left(1+\varepsilon+\cdots+\varepsilon^{n-1}\right) \delta(x) x^{n-1}$. Then $\delta^{n}\left(x^{n}\right)=\delta^{n-1}\left(\lambda_{n-1} x^{n-1}+\cdots\right)=\lambda_{n-1} \delta^{n-1}\left(x^{n-1}\right)$. In other words, $\lambda_{n-1}=\frac{\delta^{n}\left(x^{n}\right)}{\delta^{n-1}\left(x^{n-1}\right)} \in \mathbb{k}^{\times}$. Moreover, it can be deduced from this recursion that $\lambda_{n-1}=\left(1+\varepsilon+\cdots+\varepsilon^{n-1}\right) \delta(x) \in \mathbb{k}^{\times}$, for any $n \geq 1$. We conclude that $\varepsilon$ is not a root of unity.

Proposition 4.2 Let $M$ be an $A^{\delta}$-module. Then $M$ satisfies the condition (2.6). Thus, $M$ is an indecomposable $A^{\delta}$-module if and only if $A \otimes_{A^{\delta}} M$ is an indecomposable B-module. 
Proof Note that $A$ is a free right $A^{\delta}$-module with a basis $\left\{x^{i} \mid i \geq 0\right\}$ and $\delta\left(x^{n}\right)=\lambda_{n-1} x^{n-1}+\cdots$, where $\lambda_{n-1}=\left(1+\varepsilon+\cdots+\varepsilon^{n-1}\right) \delta(x) \in \mathbb{k}^{\times}$by Lemma 4.1. For any left $A^{\delta}$-module $M$, it is straightforward to check that

$$
M \rightarrow \operatorname{ker}_{t}\left(A \otimes_{A^{\delta}} M\right), m \mapsto 1 \otimes m
$$

is an $A^{\delta}$-modules isomorphism. By Theorem 2.3, we obtain that $M$ is an indecomposable $A^{\delta}$-module if and only if $A \otimes_{A^{\delta}} M$ is an indecomposable $B$-module.

Recall that $\operatorname{tor}_{T}(X)$ is a submodule of $X$, by Corollary 3.3 based on the fact that $T$ is a left Ore set.

Theorem 4.3 For any $B$-module $X$, there is a B-module isomorphism $A \otimes_{A^{\delta}} \operatorname{ker}_{t} X \cong \operatorname{tor}_{T}(X)$.

Proof Given the $\mathbb{k}$-linear map

$$
\varphi: A \otimes_{A^{\delta}} \operatorname{ker}_{t} X \rightarrow \operatorname{tor}_{T}(X), \sum_{i \geq 0} x^{i} \otimes \mu_{i} \mapsto \sum_{i \geq 0} x^{i} \mu_{i}
$$

It is easy to see that $\varphi$ is a $B$-module morphism. If $\sum_{i=0}^{n} x^{i} \otimes \mu_{i} \neq 0$, where $\mu_{n} \neq 0$, then

$$
t^{n}\left(\sum_{i=0}^{n} x^{i} \mu_{i}\right)=\sum_{i=0}^{n} t^{n} x^{i} \mu_{i}=\sum_{i=0}^{n} \delta^{n}\left(x^{i}\right) \mu_{i}=\delta^{n}\left(x^{n}\right) \mu_{n} \neq 0 .
$$

It follows that $\varphi$ is injective. The next step is to prove that $\varphi$ is surjective. Since $A$ is freely with a basis $\left\{x^{i} \mid i \geq 0\right\}$, the image of $A \otimes_{A^{\delta}} \operatorname{ker}_{t} X$ under $\varphi$ is $\sum_{i>0} x^{i} \operatorname{ker}_{t} X$. Note that $\operatorname{tor}_{T}(X)=\cup_{s \geq 1} \operatorname{ker}_{t^{s}} X$, where $\operatorname{ker}_{t^{s}} X$ is a subspace of $X$ whose elements are annihilated by $t^{s}$. We shall prove by induction on $s \geq 1$ that $\operatorname{ker}_{t^{s}} X \subseteq \sum_{i \geq 0} x^{i} \operatorname{ker}_{t} X$. If $s=1$, the inclusion $\operatorname{ker}_{t} X \subseteq \sum_{i \geq 0} x^{i} \operatorname{ker}_{t} X$ is trivial. Suppose that the inclusion $\operatorname{ker}_{t^{s}} X \subseteq \sum_{i \geq 0} x^{i} \operatorname{ker}_{t} X$ holds for $1 \leq s \leq m$. Let $\mu \in \operatorname{ker}_{t^{m+1}} X$. Then $t^{m+1} \mu=0$. By Lemma 4.1 (2), we have $t^{m+1} x=\alpha^{m+1}(x) t^{m+1}+\lambda_{m} t^{m}$, where $\lambda_{m}=\delta(x)\left(1+\varepsilon+\cdots+\varepsilon^{m}\right)$. It deduces that

$$
t^{m}\left(t x-\lambda_{m}\right) \mu=\alpha^{m+1}(x) t^{m+1} \mu=0 .
$$

Thus, by the induction hypothesis, $\left(t x-\lambda_{m}\right) \mu \in \sum_{i} x^{i} \operatorname{ker}_{t} X$. Note that $t x=\alpha(x) t+\delta(x)$. It yields that $\left(\alpha(x) t+\delta(x)-\lambda_{m}\right) \mu \in \sum_{i} x^{i} \operatorname{ker}_{t} X$. But $t^{m}(t \mu)=0$ implies that $t \mu \in \sum_{i} x^{i} \operatorname{ker}_{t} X$, and hence $\alpha(x) t \mu \in \sum_{i} x^{i} \operatorname{ker}_{t} X$. It follows that $\left(\delta(x)-\lambda_{m}\right) \mu \in \sum_{i} x^{i} \operatorname{ker}_{t} X$. We obtain that $\mu \in \sum_{i} x^{i} \operatorname{ker}_{t} X$ since $\delta(x)-\lambda_{m}=-\varepsilon \lambda_{m-1} \in \mathbb{k}^{\times}$, as required.

Denote by $B$ ( $T$-torsion) the full subcategory of $B$-Mod whose objects are $T$-torsion modules. We are now ready to state the main result, which leads to a categorical version of Kashiwara's theorem for Weyl algebras [4, Ch 18, Theorem 3.1].

Theorem 4.4 The functor $\operatorname{ker}_{t}-: B(T$-torsion $) \rightarrow A^{\delta}$-Mod gives an equivalence of categories with inverse functor $A \otimes_{A^{\delta}}$.

Proof For any object $M \in A^{\delta}$-Mod, it follows from Proposition 4.2 that $\operatorname{ker}_{t}\left(A \otimes_{A^{\delta}} M\right) \cong M$. For any object $X \in B$ (T-torsion), by Theorem 4.3, we have $A \otimes_{A^{\delta}} \operatorname{ker}_{t} X \cong \operatorname{tor}_{T}(X)=X$. It is easy to check that the two isomorphisms given above are functorial. That is, for each $X \stackrel{f}{\rightarrow} Y$ in $B$ (T-torsion) and $M \stackrel{g}{\rightarrow} N$ in $A^{\delta}$-Mod, the following two diagrams

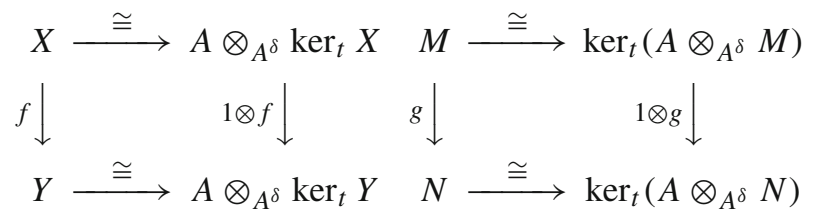

are commutative in $B\left(T\right.$-torsion) and $A^{\delta}$-Mod, respectively, as desired. 


\section{Applications to Weyl algebras}

In this section, we present a concrete application. For any integer $n \geq 1$, the $n$th Weyl algebra $A_{n}$ over a field $\mathbb{k}$ of characteristic zero is the algebra generated by $2 n$ generators $x_{1}, \ldots, x_{n}, y_{1}, \ldots, y_{n}$ with relations:

$$
\left[y_{i}, x_{i}\right]=1,\left[y_{i}, x_{j}\right]=\left[y_{i}, y_{j}\right]=\left[x_{i}, x_{j}\right]=0,
$$

for $1 \leq i, j \leq n$ and $i \neq j$, where $[a, b]:=a b-b a$. It is known that $A_{n}$ is a simple noetherian domain with a $\mathbb{k}$-basis $\left\{x_{1}^{i_{1}} \cdots x_{n}^{i_{n}} y_{1}^{j_{1}} \cdots y_{n}^{j_{n}} \mid i_{1}, \ldots, i_{n}, j_{1}, \ldots, j_{n} \in \mathbb{N}\right\}$.

The Weyl algebra $A_{n}$ can be viewed as the iterated Ore extensions

$$
A_{n}=A_{n-1}\left[x_{n}\right]\left[y_{n} ; \partial_{n}, \mathrm{id}\right],
$$

for $n \geq 1$, where $\partial_{n}$ is the differential operator with respect to the independent $x_{n}$ and $A_{0}=\mathbb{k}$. It is easy to see that the subalgebra $\operatorname{ker}\left(\partial_{n}\right)$ of $A_{n-1}\left[x_{n}\right]$ is exactly the $(n-1)$ th Weyl algebra $A_{n-1}$. In view of this, any left $A_{n-1}$-module $M$ induces a left $A_{n}$-module $A_{n-1}\left[x_{n}\right] \otimes_{A_{n-1}} M$. The following two results follow directly from Proposition 2.4 and Theorem 4.4, respectively.

Proposition 5.1 For any $A_{n-1}$-module $M, M$ is indecomposable if and only if the induced $A_{n}$-module $A_{n-1}\left[x_{n}\right] \otimes_{A_{n-1}} M$ is indecomposable.

Theorem 5.2 (Kashiwara's theorem) The functor

$$
\operatorname{ker}_{y_{n}}-: A_{n}(T \text {-torsion }) \rightarrow A_{n-1} \text {-Mod }
$$

gives an equivalence of categories with the inverse functor $A_{n-1}\left[x_{n}\right] \otimes_{A_{n-1}}$-.

We shall now investigate the relationship of Gelfand-Kirillov dimension and multiplicity between the finitely generated $A_{n-1}$-module $M$ and the induced $A_{n}$-module $A_{n-1}\left[x_{n}\right] \otimes_{A_{n-1}} M$. We begin with the introduction of the notations of Gelfand-Kirillov dimension and multiplicity for any finitely generated $A_{n}$-module. We refer to [4] for more details.

Let $\mathcal{B}=\left\{B_{k}\right\}_{k \in \mathbb{N}}$ be the Bernstein filtration of the Weyl algebra $A_{n}$ and $M$ a finitely generated $A_{n}$ module with generators $u_{1}, u_{2}, \ldots, u_{s}$. Denote by $\Gamma_{0}=\operatorname{span}\left\{u_{1}, u_{2}, \ldots, u_{s}\right\}$ and $\Gamma_{k}=B_{k} \Gamma_{0}$, for $k \geq 1$. Then $\left\{\Gamma_{k}\right\}_{k \in \mathbb{N}}$ is a good filtration of $M$ with respect to the Bernstein filtration $\mathcal{B}[4$, Ch 8, $\S 3]$. Furthermore, the graded module $\operatorname{gr}^{\Gamma} M:=\bigoplus_{k} \geqslant 0 \Gamma_{k} / \Gamma_{k-1}\left(\Gamma_{k}=\{0\}\right.$ if $\left.k<0\right)$ is generated over $S_{n}$ by the symbols $u_{1}, u_{2}, \ldots, u_{s}$, where $S_{n}=\operatorname{gr}^{\mathcal{B}} A_{n}$, which is isomorphic to the polynomial ring with $2 n$ variables over the field $\mathbb{k}_{k}$. Note that $S_{n}$ is a noetherian graded ring with $\mathbb{k}_{\mathrm{k}}$ the homogeneous component of degree 0 . This implies that $\Gamma_{k} / \Gamma_{k-1}$ is a finite dimensional $\mathbb{k}$-vector space. Therefore, $\operatorname{dim}_{\mathbb{k}}\left(\Gamma_{k} / \Gamma_{k-1}\right)<\infty$, for any $k \in \mathbb{N}$. The Hilbert polynomial of $S_{n}$-module $\mathrm{gr}^{\Gamma} M$ is given by

$$
\chi(t, \Gamma, M)=\sum_{k=0}^{t} \operatorname{dim}_{\mathbb{k}}\left(\Gamma_{k} / \Gamma_{k-1}\right)=\operatorname{dim}_{\mathbb{k}} \Gamma_{t},
$$

for $t \gg 0$. The Gelfand-Kirillov dimension $\mathrm{GK}(M)$ of $M$ is equal to the degree of the Hilbert polynomial $\chi(t, \Gamma, M)$. The multiplicity of $M$ is

$$
\mathrm{e}(M)=\mathrm{GK}(M) ! a_{\mathrm{GK}(M)},
$$

where $a_{\mathrm{GK}(M)}$ is the leading coefficient of the Hilbert polynomial $\chi(t, \Gamma, M)$. A finitely generated $A_{n}$-module is called holonomic if it is zero, or if it is of Gelfand-Kirillov dimension $n$. The number $n$ is the minimal possible Gelfand-Kirillov dimension for any non-zero finitely generated $A_{n}$-module by the Bernstein inequality.

Theorem 5.3 Let $M$ be a finitely generated $A_{n-1}$-module.

(1) $G K\left(A_{n-1}\left[x_{n}\right] \otimes_{A_{n-1}} M\right)=1+G K(M)$.

(2) $e\left(A_{n-1}\left[x_{n}\right] \otimes_{A_{n-1}} M\right)=e(M)$. 
Proof Note that the polynomial ring $\mathbb{k}\left[x_{1}\right]$ is a left $A_{1}$-module, where the actions of $x_{1}$ and $y_{1}$ on $\mathbb{k}\left[x_{1}\right]$ are given, respectively, by left multiplication and the differential operator $\partial_{1}$. Then the tensor product $\mathbb{k}\left[x_{1}\right] \otimes_{\mathbb{k}} M$ is naturally a left $A_{1} \otimes_{\mathbb{k}} A_{n-1}$-module, and so is an $A_{n}$-module by the observation of the algebra isomorphism $A_{1} \otimes_{\mathbb{k}} A_{n-1} \cong A_{n}$. By [12, Theorem 4.1], we have

$$
\mathrm{GK}\left(\mathbb{k}_{k}\left[x_{1}\right] \otimes_{\mathbb{k}} M\right)=\mathrm{GK}\left(\mathbb{k}_{k}\left[x_{1}\right]\right)+\mathrm{GK}(M)=1+\mathrm{GK}(M)
$$

and

$$
\mathrm{e}\left(\mathbb{k}_{k}\left[x_{1}\right] \otimes_{\mathbb{k}} M\right)=\mathrm{e}\left(\mathbb{k}\left[x_{1}\right]\right) \mathrm{e}(M)=\mathrm{e}(M) .
$$

Then the results follow from the following $A_{n}$-module isomorphism:

$$
\mathbb{k}\left[x_{1}\right] \otimes_{\mathbb{k}} M \rightarrow A_{n-1}\left[x_{n}\right] \otimes_{A_{n-1}} M, \sum_{i} f_{i}\left(x_{1}\right) \otimes m_{i} \mapsto \sum_{i} f_{i}\left(x_{n}\right) \otimes m_{i}
$$

As an immediate consequence of Theorem 5.3, we have the following corollary.

Corollary 5.4 Let $M$ be a finitely generated $A_{n-1}$-module. Then $M$ is a holonomic $A_{n-1}$-module if and only if $A_{n-1}\left[x_{n}\right] \otimes_{A_{n-1}} M$ is a holonomic $A_{n}$-module.

Remark 5.5 Theorem 5.3 allows us to construct holonomic $A_{n}$-modules possessing any multiplicity. More explicitly, let $M_{0}$ be a finite dimensional $\mathbb{k}$-vector space with $\operatorname{dim}_{\mathbb{k}} M_{0}=m$. Then $M_{0}$ is a finitely generated left module over $A_{0}=\mathbb{k}$. Define $A_{n}$-module $M_{n}$ recursively as $M_{n}=A_{n-1}\left[x_{n}\right] \otimes_{A_{n-1}} M_{n-1}$, for $n \geq 1$. Then $M_{n}$ is holonomic with multiplicity $m$ by Theorem 5.3.

Acknowledgments This work was supported by the NSFC (Grant No. 11471282), the NSF of the Jiangsu Higher Education Institutions of China (Grant No. 15KJB110013) and the NSF of Jiangsu Province of China (Grant No. BK20150537). We thank the referees for their careful reading of this article and for valuable comments.

Open Access This article is distributed under the terms of the Creative Commons Attribution 4.0 International License (http:// creativecommons.org/licenses/by/4.0/), which permits unrestricted use, distribution, and reproduction in any medium, provided you give appropriate credit to the original author(s) and the source, provide a link to the Creative Commons license, and indicate if changes were made.

\section{References}

1. Bavula, V.V.: The simple modules of the Ore extensions with coefficients from a dedekind ring. Comm. Algebra 27(6), 2665-2699 (1999)

2. Bavula, V.V.; van Oystaeyen, F.: Simple holonomic modules over rings of differential operators with regular coefficients of Krull dimension 2. Trans. Am. Math. Soc. 353, 2193-2214 (2001)

3. Björk, J.-E.: The global homological dimension of some algebras of differential operators. Invent. Math. 17, 67-78 (1972)

4. Coutinho, S.C.: A Primer of Algebraic D-Modules. Cambridge University Press, Cambridge (1995)

5. Goodearl, K.R.; Warfield, R.B.: An Introduction to Noncommutative Noetherian Rings, 2nd edn. Cambridge University Press, Cambridge (2004)

6. Jain, S.K.; Lam, T.Y.; Leroy, A.: Ore extensions and V-domains. Contemp. Math. 480, 263-288 (2009)

7. Jordan, D.: Finite-dimensional simple modules over skew polynomial rings. J. Pure Appl. Algebra 98, 45-55 (1995)

8. Jordan, D.: Iterated skew polynomial rings and quantum groups. J. Algebra 156(1), 194-218 (1993)

9. Jordan, D.: Iterated skew polynomial rings of Krull dimension two. Arch. Math. 61, 344-347 (1993)

10. Lam, T.Y.; Leroy, A.: Principal one-sided ideals in Ore polynomial rings. Contemp. Math. 259, 333-352 (2000)

11. Nordstrom, H.: Associated primes over Ore extensions. J. Algebra 286, 69-75 (2005)

12. Wang, Z.; Wei, J.; Li, L.: Poincaré series and an application to Weyl algebras. Acta Math. Sci. 31B(2), 459-467 (2011) 